\title{
Monostatic ultra-wideband GPR antenna for through wall detection
}

\author{
Jawad Ali ${ }^{1,2, *}$, Noorsaliza Abdullah ${ }^{1}$, Roshayati Yahya ${ }^{1}$, and Taimoor Naeem ${ }^{2}$ \\ ${ }^{1}$ Department of Communication Engineering, Faculty of Electrical and Electronic Engineering, Universiti Tun Hussein Onn \\ Malaysia (UTHM), Johor, Malaysia \\ ${ }^{2}$ Electrical Engineering Department, COMSATS Institute of Information Technology (CIIT), Lahore, Pakistan
}

\begin{abstract}
The aim of this paper is to present a monostatic arc-shaped ultra-wideband (UWB) printed monopole antenna system with 3-16 GHz frequency bandwidth suitable for through-wall detection. Ground penetrating radar (GPR) technique is used for detection with the gain of $6.2 \mathrm{~dB}$ achieved for the proposed antenna using defected ground structure (DGS) method. To serve the purpose, a simulation experiment of through-wall detection model is constructed which consists of a monostatic antenna act as transmitter and receiver, concrete wall and human skin model. The time domain reflection of obtained result is then analysed for target detection.
\end{abstract}

\section{Introduction}

Ultra-wideband (UWB) is a wireless technology, which is mainly referred to a system with very larger operating bandwidth, usually this bandwidth is considered to be greater than $500 \mathrm{MHz}$. Where, after 2002 the free localization of UWB spectrum for industrial and scientific purposes, opened new gates for the researchers to develop different technologies for the use of UWB [1]. UWB covers the frequency band ranges from 3.1$10.6 \mathrm{GHz}$ with $7.5 \mathrm{GHz}$ bandwidth, there are several advantages of this very large bandwidth as it consumes less power, have low implementation cost, with high data rate and time-resolution, also have the capability of obstacle penetration [2]. Such advantages widen the number of prominent applications in the field of communications such as wireless local area network (WLAN), wireless body area network (WBAN), WiMAX, wireless personal area network (WPAN), also in the field of radar like ground penetrating radars (GPR), imaging and positioning [3].

UWB technology, from sometime has been in use for many GPR applications [4]. GPR have a number of applications in the field of engineering for military and civilian applications like the detection of landmines, through-wall imaging, in remote sensing techniques such as non-destructive testing of concrete and detection of trapped people under debris or in the opaque environment [5-6]. For the implementation of UWB technology specifically in the applications of GPR different types of antenna like a horn, bow tie, tapered slot, patch, spiral, and dipole antennas have been designed previously. These type of antennas are considered for their high gain, design complexity, directivity and frequency bandwidth which are the basic requirements in antenna design for GPR applications [7-
9]. In this paper, an arc-shaped printed monopole UWB antenna with defected ground structure (DGS) is designed that have the ability to operate from $3-16 \mathrm{GHz}$ bandwidth with the gain of around $6 \mathrm{~dB}$ and GPR model for through-wall detection prototype is simulated using CST-MWS, the reflection of the target is then computed and demonstrated using MATLAB.

The remainder of the paper is organized as follows: in Section 2 antenna design and complete simulation model for through-wall imaging by using GPR technology is presented, the discussion about the analysis of obtained results is in Section 3. Finally, Section 4 summarizes the complete discussion in the form of conclusion.

\section{Design and modeling}

This section is further categorized into two sub-sections. Section 2.1 is about antenna design for ultra-wideband (UWB) ground penetrating radar (GPR) use in detection, while Section 2.2 discusses the simulation model designed using CST-MWS for experimental purpose.

\subsection{Proposed antenna design}

For the implementation of ultra-wideband (UWB) antenna ground penetrating radar (GPR) to address the through wall penetration, an arc shape printed monopole antenna with defected ground structure (DGS) is introduced in this paper. The arc shape design of the antenna is achieved by cutting a circular slot towards the minor axis of elliptical shape patch with microstrip line feed. The proposed UWB arc antenna design is shown in Fig. 1, the dimensions of the substrate, microstrip line shows that designed antenna also have a compact shape.

* Corresponding author: engrjawad.ali@outlook.com 
The main challenges in designing UWB compact shape antenna is the bandwidth matching at low frequencies [10] of the band from 3.1-10.6 GHz, to achieve the matching over complete band a modified half ground is introduced in the design. But the gain obtained as a result was very low, which was again another challenge because for GPR applications the gain should be nominal. For the gain to be around $6 \mathrm{~dB}$ DGS [11] technique is used on the ground and as a result of this technique, slots are introduced at the different locations on the ground as shown in Fig. 1. The substrate material used in this design is FR-4 with $\mathrm{Er}=4.2$. Table 1 shows the dimensions along with the substrate material, ground, and the major and minor axis of the elliptical arc.
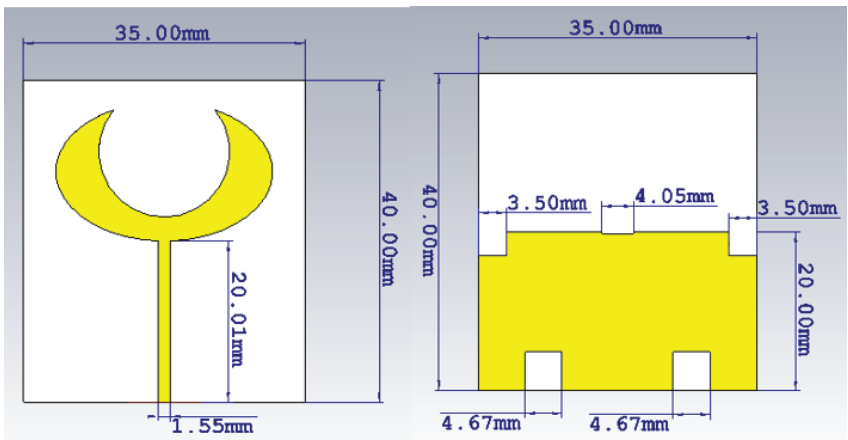

Fig. 1. Arc Antenna Design

Table 1. Design Parameters of Arc Antenna

\begin{tabular}{|l|l|l|}
\hline Parameter Name & Material & Dimension (mm) \\
\hline Major arc axis & Copper & 27 \\
\hline Minor arc axis & Copper & 17.28 \\
\hline Substrate (LxW) & FR-4 & $40 \times 35$ \\
\hline Ground (LxW) & Copper & $20 \times 17.5$ \\
\hline
\end{tabular}

\subsection{Simulation model}

The antenna discussed in Section 2.1 is specifically designed using CST-MWS for through-wall detection application using ground penetrating radar (GPR) technique in order to enhance the detection capability of compact antenna design. The proposed through-wall detection experiment is designed and simulated in CSTMWS environment. Fig. 2 shows the prototype of complete designed model where monostatic antenna used for transmission and reception is placed at some distance from $10 \mathrm{~cm}$ thick and 1 year old concrete wall, and human skin targeted model is on the other side of the wall. The distance from antenna to the wall is measured as D_Wall whereas between wall and target it is computed as D_Target. The dielectric constant of wall throughout the ultra-wideband (UWB) is $\varepsilon_{\mathrm{r}}=5.46$, while for skin it varies from higher to lower frequencies with the value $\varepsilon_{\mathrm{r}}=26-36$. The reflection based results of the complete model will be discussed in Section 3.

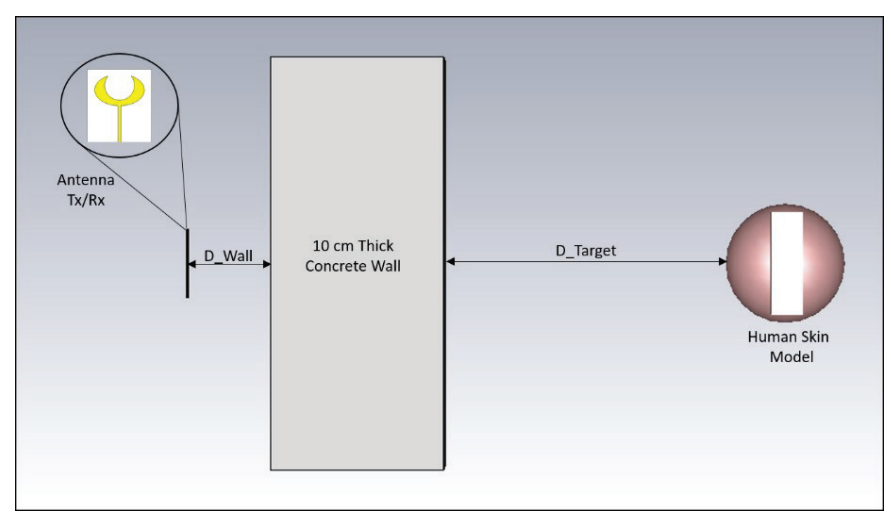

Fig. 2. Through Wall Detection Simulated Prototype

\section{Results and discussions}

The proposed antenna is simulated using CST-MWS environment and the obtained results are shown in Fig. 3. The return loss $\left(\mathrm{S}_{11}\right)$ in Fig. 3a shows the result is significant as it covers the complete ultra-wideband (UWB), even the simulated band is from $3-16 \mathrm{GHz}$ which is more than the actual band from 3.1-10.6 GHz. The voltage standing wave ratio (VSWR) of the proposed design is also less than 2 as shown in Fig. 3b, this VSWR value shows that the designed antenna is radiating from 3-16 GHz without any interference. Fig. $3 c$ demonstrates the information about the gain of the proposed antenna is $6.21 \mathrm{~dB}$, this value of gain is achieved by introducing slots in the ground plane using defected ground structure (DGS) method. The obtained gain is suitable for through-wall detection as its radiation is also directional.

After the successful simulation of proposed antenna design, a complete monostatic ground penetrating radar (GPR) experiment is performed for detection purpose as discussed in Section 2.2. The obtained results from experimentation are further analyzed in the time domain using inverse Fourier transformation technique to study the difference between the reflections from antenna, wall, and target.

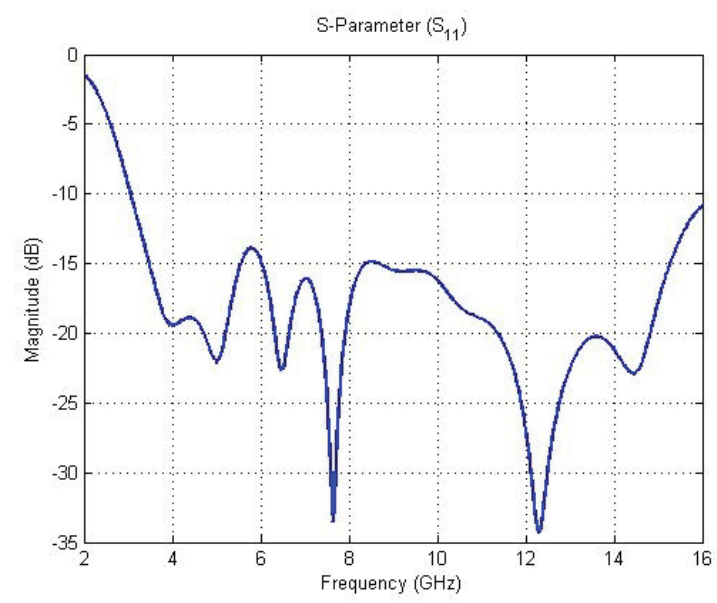

(a) 


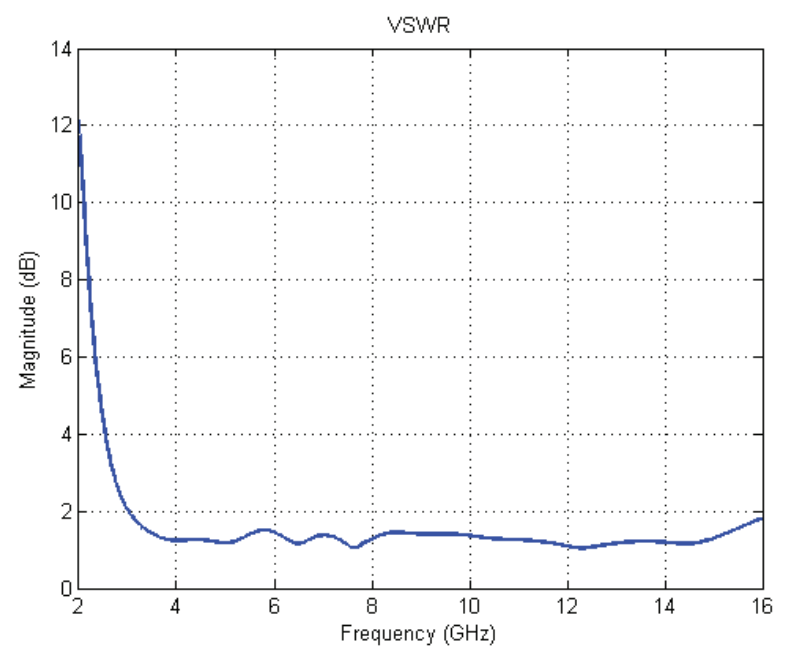

(b)

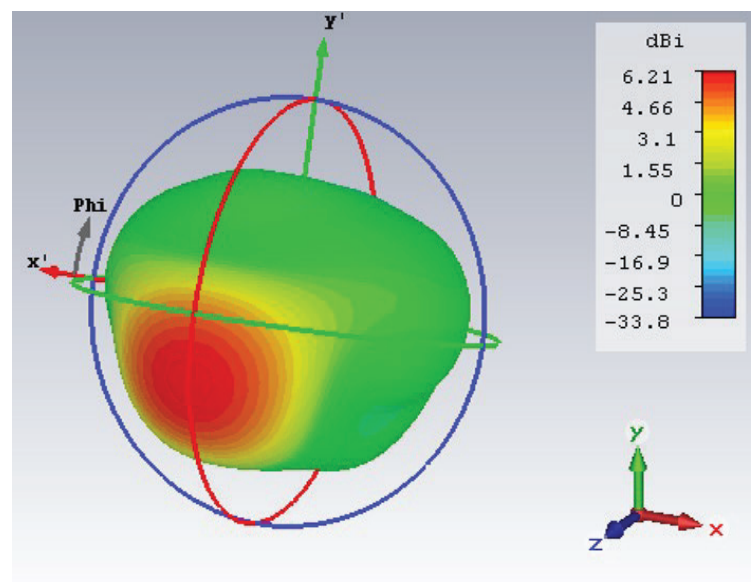

(c)

Fig. 3. Simulated Results of Arc Antenna (a) S-Parameter Result from 3-16 GHz (b) VSWR $<2$ for proposed antenna (c) 3D Simulated Antenna Gain

Fig. 4 shows the time reflection results of a monostatic antenna system using GPR technique, it mainly consists of two times interval resolutions the first with dashed line is only from antenna and wall while the second with the continuous line is the reflection from antenna, wall, and target. From the figure, it is observed that the reflection from the wall is received at $0.33 \mathrm{~ns}$, while the reflection before $0.33 \mathrm{~ns}$ is considered to be the antenna coupling. The second time interval shows a peak at $2.8 \mathrm{~ns}$, this peak is the obvious reflection from the targeted object behind the wall. From different time intervals $(t)$, the depth resolution $(d)$ of the target and the wall can also be determined using [12]:

$$
\begin{aligned}
& d=v \frac{t}{2} \\
& v=\frac{c}{\sqrt{\varepsilon_{r}}}
\end{aligned}
$$

Where $(v)$ is the propagation velocity in terms of speed of light $(c)$ and dielectric constant $\left(\varepsilon_{\mathrm{r}}\right)$.

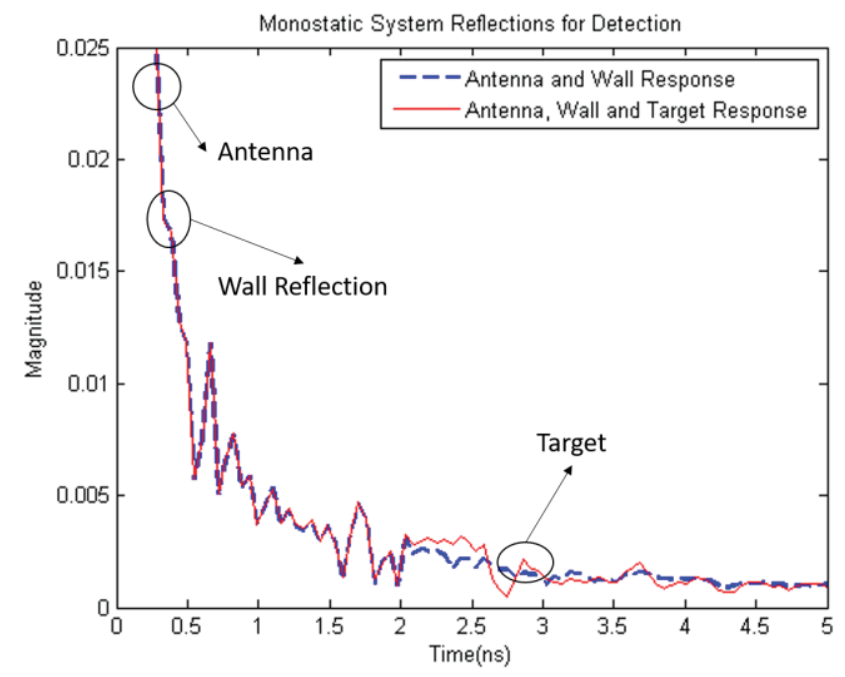

Fig. 4. Antenna, Wall and Target Reflections

It has also been observed that there is an insignificant difference between antenna and wall reflections, the reason of this insignificance is aliasing that occurs mainly due to the inadequate sampling rate [13].

\section{Conclusions}

A complete monostatic ultra-wideband (UWB) model for through-wall detection is discussed during the paper. The proposed arc shape antenna having defected ground structure (DGS) with the gain of $6.2 \mathrm{~dB}$ and $13 \mathrm{GHz}$ bandwidth is designed using FR-4. This antenna is then modeled and simulated with wall and human skin structure as a targeted object for detection purpose. Later the results are analyzed to see the time domain reflections obtained from simulation. This demonstration hence makes the UWB monostatic antenna one of the potential candidates for detection applications.

The authors would like to thanks ORICC Universiti Tun Hussein Onn Malaysia (UTHM) for supporting this work.

\section{References}

1. M. M. Islam, M. Samsuzzaman, M. R. I. Faruque, Micro. and Opt. Tech. Lett. 57, 1470-1475 (2015) .

2. D. Valderas, X. Chen, C. Ling, J. I. Sancho, D. Puente, Ultrawideband antennas: Design and applications, Imperial College Press, London, (2010)

3. F. B. Zarrabi, Z. Mansouri, N.P. Gandji, H. Kuhestani, AEU-Int. J of Electro. and Comm. 70, 64-69 (2016)

4. A. Elboushi, A. R. Sebak, T. Denidni, ACES, 28, 411-418 (2013)

5. C. Warren, A. Giannopoulos, IEEE J of Sel. Top. in Appl. Earth Obs. and Rem. Sens. 9, 29-36 (2016). 
6. C. Ozdemir, B. Yilmaz, S. I. Keceli, H. Lezki, O. Sutcuoglu, 15th International Radar Symposium (IRS), Gdansk, (2014).

7. A. Ahmed, Y. Zhang, D. Burns, D. Huston, T. Xia, IEEE Geosci. and Rem. Sens. Lett. 13, 92-96, (2016).

8. E. A. Etellisi, M. A. Elmansouri, D. S. Filipovic, IEEE Trans. on Ant. and Prop. 64, 6-15, (2016).

9. E. A. Etellisi, M. A. Elmansouri, D. S. Filipovic, IEEE International Symposium on Antennas and Propagation (APSURSI), Fajardo,( 2016).

10. S. Koziel, A. Bekasiewicz, IEEE Ant. and Wire. Prop. Lett. 15, 750-753, (2016).

11. R.A. Pandhare, P.L. Zade, M.P. Abegaonkar, Engg. Sci. and Tech., an Int. J. 19, 1360-1367, (2016).

12. D. J. Daniels, Ground Penetrating Radar (The Institution of Engineering and Technology, 2004)

13. X. Zeng, A. Fhager, M. Persson, P. Linner and H. Zirath, IEEE Trans. on Ant. and Prop. 59, 42794285, (2011). 